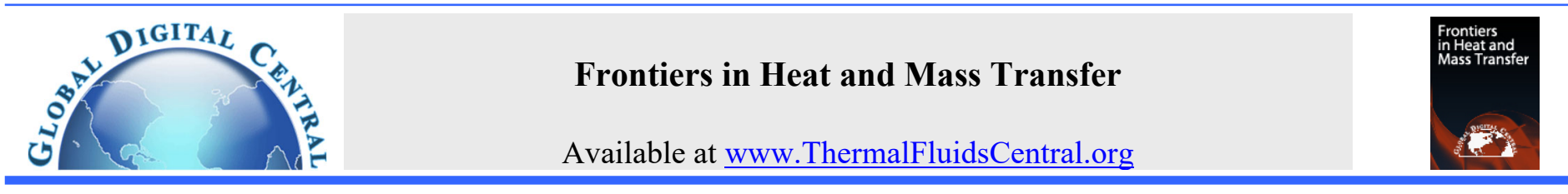

\title{
NUMERICAL SOLUTION OF THE EFFECTS OF HEAT AND MASS TRANSFER ON UNSTEADY MHD FREE CONVECTION FLOW PAST AN INFINITE VERTICAL PLATE
}

\author{
D. Santhi Kumari ${ }^{a,}$, Venkata Subrahmanyam Sajja ${ }^{a}$, P. M. Kishore ${ }^{\text {b, }}$ \\ ${ }^{a}$ Department of Mathematics, Koneru Lakshmaiah Education Foundation, Guntur-522502, A.P., India. \\ ${ }^{b}$ Department of Mathematics, Geethanjali Institute of Science and Technology, Nellore, A.P., India.
}

\begin{abstract}
This study attempts to explore a qualitative analysis of the effects of Soret on an unsteady magnetohydrodynamics free convection flow of a chemically reacting incompressible fluid past an infinite vertical plate embedded in a porous medium taking the source of heat and thermal radiation into account as well as viscous dissipation. The central equations are scrupulously converted into sets of coupled nonlinear partial differential equations for providing logical solutions. The method of Galerkin finite element is used considering appropriate boundary conditions for diverse physical metrics and then numerically analyzed employing MATLAB. A significant change in velocity, temperature, concentration profiles is observed for various values of Prandtl number, Grashof number, Eckert number, Soret number. It is noticed that the velocity profile enhances by enhancing the values of porous medium as well as it decreases when ' $M$ ' and Prandtl number increases. The temperature profile decreases as for the increasing vales of heat source parameter and also the concentration profile increases as Soret number increases.

Keywords: Galerkin Method; Magnetohydrodynamics; Chemical Reaction; Viscous Dissipation; Heat Source; Soret Effect
\end{abstract}

\section{INTRODUCTION}

The natural physical process of convection flow has been gaining increasing research attention owing to its increased utilization in diverse physical, chemical and engineering applications. Natural convection is a physical process of transferring heat and mass among the fluids initiated by the variation in the concentration of both the temperature as well as the species resulting in the differences in density and encouraging the forces of buoyancy to operate on the fluids. The natural convection of the Nano fluids from a vertical accelerated plate in the presence of the radiation flux and magnetic field is observed in this study by Astuti et al.

(2019). Nature contains enormous quantities of such flows. This process of convection is studied in depth due to its extensive free applications in the environments of geophysical sciences and engineering. Room heating inside the buildings utilizing radiators could well illustrate the application of convection for transferring heat. Buoyancy or decrease in gravity Middleman (1998), Rubin et al. (2001) is the prime factor causing these natural flows of convection due to the variation in temperature resulting in corresponding differences in fluid density, including liquids and gases. It has been identified that the flows induced by buoyancy in the fluid-saturated porous media have been extensively employed in a wide variety of applications of thermal engineering applications and domains such as geothermic system spring water pollution, exchangers of heat, thermal insulation, piling up of nuclear effluence, packed bed catalytic reactors, cooling of electronic devices, the security of energy systems, atmosphere and oceanic circulation.
Several researchers are drawn towards the field of Magnetohydrodynamics due to its vast and varied applications in the realms of geophysical science and astrophysics. MHD is largely utilized in the advanced studies on the astronomical matter, solar and stellar structures, propagation of radio through the medium of ionosphere, etc. Several applications associated with oil extraction, recovery of heat, flow-through devices of filtration, and storage of thermal energy largely make use of the applications based on the progression of convection through permeable media, besides MHD bearings and MHD pumps. The modern times witness an enhanced focus on this heat transfer process through convection due to its ever-enhancing prominence in diverse technological innovations and applications such as fiber and granular insulation, electronic system cooling, cool combustors, and porous material regenerative heat exchangers, geo-thermal reservoirs, and drying the porous solids. This feature of concurrent heat and mass transfer from entirely diverse geometrics embedded in porous media encompasses a range of engineering and geophysical science applications as have been mentioned.

Soundalgekar and Wavre (1977) have explored an unstable free convection flow of convection past an infinite vertical plate that contains continuous suction and shifting of mass. Agrawal H et al. (1984) furnish the influence that Hall currents could have on hydro-magnetic free convection with the transfer of mass in an extremely rotating fluid. Jang and $\mathrm{Ni}(1989)$ have analyzed transient-free convection with a transfer of mass from an equal vertical plate embedded in a medium that has an extremely permeable nature. Jha (1991) studied the characteristics of the free convection of MHD and the subsequent flow of transfer of mass through a porus medium. Prabhakar Reddy (2020) has studied how the transfer of mass impacts the free convective flow on an unsteady MHD

\footnotetext{
${ }^{*}$ Research Scolar. Presently at Department of Science \& Humanities, Rise Krishna Sai Group of Institutions, Ongole, Andhra Pradesh, India.

${ }^{\dagger}$ Corresponding author. Email: shanti4257@gmail.com.
} 
containing incompressible viscous dissipative fluid past an infinite vertical porous plate. MHD flow of carreau nano fluid explores using CNT over a nonlinear elongated sheet is studied by Nagalakshmi et al. (2020).

The contribution of radiation becomes important for air in instances where the wall temperature ranges between the var6000 $-10,000 \mathrm{~K}$. Such instances occur when vehicles in space attempt reentry into the atmosphere. Korycki (2006) illustrates this radioactive heat transfer as a vital and fundamental phenomenon that exists in all sensible engineering processes as can be found in radiation within the buildings, foundry engineering, and set processes, die formation, chemical engineering, and diverse composite structures applied in manufacturing.

Thermal radiation has become an integral and potential component in the field of fluid dynamics emerging as one of the premium branches in engineering sciences with its prominent aspect of fusing diverse scenarios in mechanical, chemical, aerospace, environmental, stellar energy and hazards engineering. Bhaskara Reddy and Bathaiah (1981) \& (1982) have analyzed the MHD free streamline flow of convection of an incompressible elastic fluid. Further, they have studied the combined and strained flow of convection through two parallel porous walls. Elabashbeshy (1997) has studied the transfer of mass and heat on a vertical plate along with magnetic flux. Samad, Karim, and Muhammad (2010) have made numerical computations to estimate the influence of the thermal radiation on steady MHD free convection flow considering the diffusion approximation of Rosseland. Loganathan and Arasu (2010) have analyzed the results of the deposition of thermophoresis on non-Darcy MHD combined convective transfer of mass and heat past a porous wedge while suction or injection is present.

This paper attempts to examine, the effects of transfer of mass and heat of free convection flow- considering the viscous dissipation of a fluid past an infinite vertical porous plate embedded in a permeable medium when a homogeneous magnetic field is present. Partial differential equations of coupled non-linear systems govern the principles of the problem with inbuilt complexities to obtain precise solutions. Hence, the method of Galerkin finite element, with its computational cost efficiency, has been employed for obtaining the solutions. The consequent changes and comportment of diverse aspects such as the concentration velocity, temperature, Sherwood and Nusselt numbers have been comprehensively focused for observing the possible changes in the governing framework and metrics.

Crepeau and Clarksean (1997) have considered the classical drawback in instances of natural convection flow from an equal vertical plate along with the term of supplementary heat generation within the equation of energy. They found out that there must be exponential decay in internal heat generation with the classical variable of similarity for finding a real similar solution. Reddy and Rao (2011) examined the Soret effect on unstable MHD free convection transfer flow of mass and heat past an infinite vertical permeable plate in conjunction with the Hall current, heat source, and consequent thermal radiation. Pal and Talukdar (2012) have detailed the impact that the thermal radiation and the chemical reaction of first-order could have on unstable MHD convective transfer flow of mass and heat along with the Soret effect of a viscous fluid past a semi-infinite vertical flat plate when heat source and oscillatory suction are present.

Chemical reaction processes are both uniform and heterogeneous depending on the location of their occurrences such as an interface or single section volume. Gehart et al. (1971) have studied how foreign mass can impact the free flow of convection beyond a semi-infinite vertical plate. The far-off mass existing either in water or in the air can cause significant chemical process. In general, the reaction rate of chemical processes corresponds to the concentration levels of the species. For example, convection associated with the internal generation of heat has a pivotal role to play in the framework of the method of complete heat transfer i.e., the acquisition of metal waste from the utilized fuel and the processes of thermal combustion activity.
Alim et al. (2008) have investigated the impact of viscous dissipation over natural flow of convection along a sphere having heat loss of radiation, while Salina et al. (2010) have examined the effect of viscous dissipation over natural convection flow of convection along a sphere with the generation of heat. Alam et al. (2007) have examined the impact of viscous dissipation on MHD natural convection flow on a sphere besides heat generation.

This paper attempts to examine, the effects of transfer of mass and heat of free convection flow- considering the viscous dissipation of a fluid past an infinite vertical porous plate embedded in a permeable medium when a homogeneous magnetic field is present. Partial differential equations of coupled non-linear systems govern the principles of the problem with inbuilt complexities to obtain precise solutions. Hence, the method of Galerkin finite element, with its computational cost efficiency, has been employed for obtaining the solutions. The consequent changes and comportment of diverse aspects such as the concentration velocity, temperature, Sherwood and Nusselt numbers have been comprehensively focused for observing the possible changes in the governing framework and metrics.

\section{MATHEMATICAL ANALYSIS}

Our purpose in this paper is to study the unstable spontaneous flow of convection considering the viscous dissipation of a fluid which has the properties of electric conduction and hydro-magnetic radiation, past on infinite heated vertical plate which has been embedded in a porous medium. Fig. 1 represents the physical model including the coordinate system. The $x^{\prime}$ - axis is considered here for alongside the vertical plate, while $y^{\prime}$ - axis for normal to the plate. At the point of

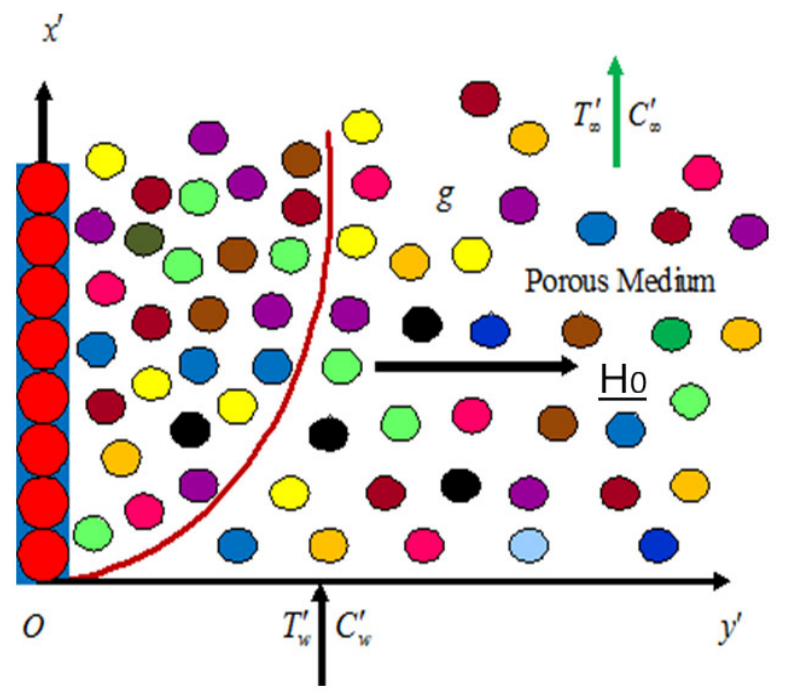

Fig.1 Schematic Diagram

time $t^{\prime}=0$, the temperatures of $T^{\prime}$ and $T_{\infty}^{\prime}$ have been steadily maintained for the plate as well as the fluid respectively and presumed to be identical. Similarly, it has been assumed that concentration levels of pertaining to the species, $C_{w}^{\prime}$ at the plate and $C_{\infty}^{\prime}$ all through the fluid, to be identical. The plate's temperature at time $t^{\prime}<0$, which has been hitherto maintained as constant, now is altered resulting in the flow of convection currents very near to the plate. Consequently, the supply of mass to the plate is at a constant rate, while the functions of time $t^{\prime}$ and

$y^{\prime}$ in this scenario, become the flow variables.

We now consider flow of an incompressible fluid making the following assumptions.

i) All the fluid properties except the density in the buoyancy force term are constant. 
ii) A homogenous magnetic field with an intensity of $H_{0}$ is executed diagonally to the plate.

iii)The magnetic field which has been thus induced is ignored since the flow's magnetic Reynolds number is taken to be very inconsequential.

iv) $\bar{E}=0$ the electric field is zero.

v) The Boussinesq approximation is applied.

vi) The Hall effect of magnetohydrodynamics and magnetic dissipation (Joule heating of the fluid) are neglected.

vii) $\mathrm{T}_{\mathrm{w}},>\mathrm{T}_{\infty}$

viii) The heat source $Q^{*}$ is of the type $Q^{*}=Q_{0}\left(T_{\infty}^{\prime}-T^{\prime}\right)$.

ix) The concentration of the diffusing species in the binary mixture is assumed to be very small in comparison with the other chemical species Dufour effect is negligible.

$\mathrm{x})$ The chemical reaction is considered to be homogeneous and of first order.

xi) Also, no applied or polarized voltages are assumed to exist, so that the effect of polarization of fluid is negligible.

With the foregoing assumptions and under the usual boundary layer approximations, unsteady flow is governed by the following partial differential equations

Continuity Equation:

$$
\frac{\partial v^{\prime}}{\partial y^{\prime}}=0
$$

\section{Momentum Equation:}

$$
\frac{\partial u^{\prime}}{\partial t^{\prime}}=v \frac{\partial^{2} u}{\partial y^{\prime 2}}+g \beta\left(T^{\prime}-T_{\infty}^{\prime}\right)+g \beta^{*}\left(C^{\prime}-C_{\infty}^{\prime}\right)-\frac{\sigma \mu_{e}^{2} H_{0}^{2} u^{\prime}}{\rho}-\frac{v u^{\prime}}{K^{\prime}}
$$

\section{Energy Equation:}

$\rho C_{P} \frac{\partial T^{\prime}}{\partial t^{\prime}}=k \frac{\partial^{2} T^{\prime}}{\partial y^{\prime 2}}-\frac{\partial q_{r}}{\partial y^{\prime}}+\mu\left(\frac{\partial u^{\prime}}{\partial y^{\prime}}\right)^{2}-Q_{0}\left(T^{\prime}-T_{\infty}{ }^{\prime}\right)$

\section{Concentration Equation:}

$$
\frac{\partial C^{\prime}}{\partial t^{\prime}}=D_{M} \frac{\partial^{2} C^{\prime}}{\partial y^{\prime 2}}-K_{r}^{\prime}\left(C^{\prime}-C_{\infty}^{\prime}\right)+\frac{D_{M} K_{T}}{T_{m}} \frac{\partial^{2} T^{\prime}}{\partial y^{\prime 2}}
$$

The boundary conditions for the fields of velocity, temperature, and concentration respectively are as follows:

$$
\left.\begin{array}{l}
t^{\prime} \leq 0, T^{\prime}=T_{\infty}^{\prime}, C^{\prime}=C_{\infty}^{\prime} \quad \forall y^{\prime} \\
u^{\prime}=0, T^{\prime}=T_{w}^{\prime}, C^{\prime}=C_{w}^{\prime} \quad \text { at } y^{\prime}=0 \\
u^{\prime}=0, T^{\prime} \rightarrow T_{\infty}^{\prime}, C^{\prime} \rightarrow C_{\infty}^{\prime} \quad \text { as } y^{\prime} \rightarrow \infty
\end{array}\right\}
$$

Where $u^{\prime}$ is the velocity along the $x^{\prime}$ - axis, $v^{\prime}$ is the kinematic coefficient of viscosity, $\mathrm{g}$ is the acceleration due to gravity, $\beta$ is the coefficient of volume expansion for the heat transfer, $\beta^{*}$ is the volume coefficient of expansion with species concentration, $T$ ' is the fluid temperature, $T_{\infty}^{\prime}$ is the fluid temperature at infinity, $T_{w}^{\prime}$ and $C_{w}^{\prime}$ are the wall dimensional temperature and concentration respectively. $C^{\prime}$ is the species concentration, $C_{\infty}^{\prime}$ is the species concentration at infinity, $D_{M}$ is the chemical molecular diffusivity, $k_{1}$ is the mean absorption coefficient, $K^{\prime}$ is the constant permeability of the medium, $\mu$ is the coefficient of viscosity, $C_{p}$ is the specific heat at constant pressure, $\rho$ is the density of fluid, $K_{r}^{\prime}$ - the chemical reaction parameter and $\mathrm{t}$ is the time. Equation (1) gives $v^{\prime}=-v_{0}\left(v_{0}>0\right)$
Where $v_{0}$ is the constant suction velocity with the negative sign implying it in the direction of the plate.

Employing the method of Roseland approximation, the term for radiative

heat flux is depicted by $q_{r}=-\frac{4 \sigma}{3 k_{1}} \frac{\partial T^{\prime 4}}{\partial y^{\prime}}$

where $\sigma=5.67 \times 10^{-8} \mathrm{~W} / \mathrm{m}^{2} \mathrm{~K}^{4}$ is the Stefan- Boltzmann constant and $k_{1}$ is the Roselan mean absorption coefficient. Here, it has been assumed that the variations in the temperature in the flow are insignificant enough that $T^{1^{4}}$ could be expressed as the temperature's linear function. This is achieved through the expansion of $T_{\infty}^{\prime}$ a Taylor series and ignoring the terms of a greater order. Thus

$T^{\prime 4} \cong 4 T_{\infty}^{\prime 3} T^{\prime}-3 T_{\infty}^{\prime}$

Then, by the application of (5) and (6) in equation (2), can be reduced as given below:

$\rho C_{P} \frac{\partial T}{\partial t^{\prime}}=k \frac{\partial^{2} T^{\prime}}{\partial y^{\prime 2}}+\frac{16 \sigma T_{\infty}^{\prime{ }^{\prime}}}{3 k_{1}} \frac{\partial^{2} T^{\prime}}{\partial y^{\prime 2}}+\mu\left(\frac{\partial u^{\prime}}{\partial y^{\prime}}\right)^{2}$

The non-dimensional quantities mentioned below are introduced, so that the governing equations and boundary condition can be rendered in dimensionless from:

$$
\begin{aligned}
& M=\frac{\sigma \mu_{0}^{2} H_{0}^{2} v}{\rho v_{0}^{2}}, u=\frac{u^{\prime}}{u_{0}}, y=\frac{y^{\prime} v_{0}}{V}, t=\frac{t^{\prime} v_{0}^{2}}{V}, \theta=\frac{T^{\prime}-T_{\infty}^{\prime}}{T_{w}^{\prime}-T_{\infty}^{\prime}}, \\
& C=\frac{C^{\prime}-C_{\infty}^{\prime}}{C_{w}^{\prime}-C_{\infty}^{\prime}}, P_{r}=\frac{\mu c_{p}}{k}, G_{m}=\frac{g \beta^{*}\left(C_{w}^{\prime}-C_{\infty}^{\prime}\right) V}{u_{0} v_{0}^{2}}, K_{r}=\frac{K_{r}^{\prime} v}{v_{0}^{2}}, \\
& S_{c}=\frac{v}{D_{M}}, E_{c}=\frac{u_{0}^{2}}{C_{P}\left(T_{w}^{\prime}-T_{\infty}^{\prime}\right)}, R=\frac{4 \sigma T_{\infty}^{\prime 3}}{k k_{1}}, S_{r}=\frac{D_{M} K_{T}}{v T_{m}\left(C_{w}^{\prime}-C_{\infty}^{\prime}\right)} \\
& G_{r}=\frac{v g \beta\left(T_{w}^{\prime}-T_{\infty}^{\prime}\right)}{u_{0} v_{0}^{2}}, C_{f}=\frac{\tau^{\prime} \omega}{\rho U_{0} v}=\left(\frac{\partial u}{\partial y}\right)_{y=0}, S=\frac{Q_{0}}{\rho C_{P} v},
\end{aligned}
$$

In the context of (8), equations (1), (3), and (7) have been reduced to the non-dimension form as given hereunder:

$$
\begin{aligned}
& \frac{\partial u}{\partial t}=G_{r} \theta+G_{m} C+\frac{\partial^{2} u}{\partial y^{2}}-\left(M+\frac{1}{K}\right) u \\
& \frac{\partial \theta}{\partial t}=\frac{1}{P_{r}}\left(1+\frac{4 R}{3}\right) \frac{\partial^{2} \theta}{\partial y^{2}}+E_{c}\left(\frac{\partial u}{\partial y}\right)^{2}-S \theta \\
& \frac{\partial C}{\partial t}=\frac{1}{S_{c}} \frac{\partial^{2} C}{\partial y^{2}}-K_{r} C+S_{r} \frac{\partial^{2} \theta}{\partial y^{2}}
\end{aligned}
$$

And these corresponding boundary conditions are

$$
\left.\begin{array}{l}
t \leq 0: u=0, \theta=0, C=0 \forall y \\
t \succ 0: u=0, \theta=1, C=1 \text { aty }=0 \\
u \rightarrow 0, \theta \rightarrow 0, C \rightarrow 0 \text { asy } \rightarrow \infty
\end{array}\right\}
$$

\section{NUMERICAL TECHNIQUE}

The technique of finite element has been employed for solving the issues of non-dimensional momentum as well as the equations of energy (10), (11) and (12) in addition to the imposed conditions of the boundary (13). In the context of the differential equations, the Galerkin expression (10) becomes 
$\int_{y_{j}}^{y_{k}}\left\{N^{T}\left[\frac{\partial^{2} u^{(e)}}{\partial y^{2}}-\frac{\partial u^{(e)}}{\partial t}-R u^{(e)}+P\right]\right\} d y=0$

Where $N^{T}=\left[\begin{array}{ll}N_{j} & N_{k}\end{array}\right]^{T}=\left[\begin{array}{l}N_{j} \\ N_{k}\end{array}\right]$

$R=M+\frac{1}{K}, P=(G r) \theta+\left(G_{m}\right) C ;$

Let us assume the linear piece-wise approximation solution to be $u^{(e)}=N_{j}(y) u_{j}(t)+N_{k}(y) u_{k}(t)=N_{j} u_{j}+N_{k} u_{k}$

Then, the equation of element is represented as

$\int_{y_{j}}^{y_{k}}\left\{\left[\begin{array}{cc}N_{j}^{\prime} N_{j}^{\prime} & N_{j}^{\prime} N_{k}^{\prime} \\ N_{j}^{\prime} N_{k}^{\prime} & N_{k}^{\prime} N_{k}^{\prime}\end{array}\right]\left[\begin{array}{c}u_{j} \\ u_{k}\end{array}\right]\right\} d y+\int_{y_{j}}^{y_{k}}\left\{\left[\begin{array}{cc}N_{j} N_{j} & N_{j} N_{k} \\ N_{j} N_{k} & N_{k} N_{k}\end{array}\right]\left[\begin{array}{c}\dot{u_{j}} \\ \dot{u_{k}}\end{array}\right]\right\} d y$

$+R \int_{y_{j}}^{y_{k}}\left\{\left[\begin{array}{ll}N_{j} N_{j} & N_{j} N_{k} \\ N_{j} N_{k} & N_{k} N_{k}\end{array}\right]\left[\begin{array}{l}u_{j} \\ u_{k}\end{array}\right]\right\} d y=p \int_{y_{j}}^{y_{k}}\left[\begin{array}{l}N_{j} \\ N_{k}\end{array}\right] d y$

Where prime and dot represent differentiation w.r.to ' $y$ ' and ' $t$ ' respectively. On simplification, we derive

$\frac{1}{l^{(e)^{2}}}\left[\begin{array}{cc}1 & -1 \\ -1 & 1\end{array}\right]\left[\begin{array}{l}u_{j} \\ u_{k}\end{array}\right]+\frac{1}{6}\left[\begin{array}{ll}2 & 1 \\ 1 & 2\end{array}\right]\left[\begin{array}{c}\dot{u}_{j} \\ \dot{u_{k}}\end{array}\right]+\frac{R}{6}\left[\begin{array}{ll}2 & 1 \\ 1 & 2\end{array}\right]\left[\begin{array}{l}u_{j} \\ u_{k}\end{array}\right]=\frac{P}{2}\left[\begin{array}{l}1 \\ 1\end{array}\right]$ Where

$l^{(e)}=y_{k}-y_{j}=h$

For obtaining the differential equation at the knot $x_{i}$, we have written the element equations for the two elements $y_{i-1} \leq y \leq y_{i}$ and $y_{i} \leq y \leq y_{i+1}$. Assembling these two, we get

$\frac{1}{l^{(e)^{2}}}\left[\begin{array}{ccc}1 & -1 & 0 \\ -1 & 2 & -1 \\ 0 & -1 & 1\end{array}\right]\left[\begin{array}{c}u_{i-1} \\ u_{i} \\ u_{i+1}\end{array}\right]+\frac{1}{6}\left[\begin{array}{ccc}2 & 1 & 0 \\ 1 & 4 & 1 \\ 0 & 1 & 2\end{array}\right]\left[\begin{array}{c}\bullet \\ u_{i-1} \\ \dot{u_{i}} \\ \dot{u_{i+1}}\end{array}\right]+\frac{R}{6}\left[\begin{array}{ccc}2 & 1 & 0 \\ 1 & 4 & 1 \\ 0 & 1 & 2\end{array}\right]\left[\begin{array}{c}u_{i-1} \\ u_{i} \\ u_{i+1}\end{array}\right]=\frac{P}{2}\left[\begin{array}{l}1 \\ 2 \\ 1\end{array}\right]$

The row equation of the knot ' $i$ ', is represented as

$\frac{1}{l^{(e)^{2}}}\left[-u_{i-1}+2 u_{i}-u_{i+1}\right]+\frac{1}{6}\left[\dot{u}_{i-1}+4 \dot{u}_{i}+\dot{u}_{i+1}\right]+\frac{R}{6}\left[u_{i-1}+4 u_{i}+u_{i+1}\right]=P$

On the application of the method of Crank-Nicholson to the above equation, we derive

$$
A_{1} u_{i-1}^{n+1}+A_{2} u_{i}^{n+1}+A_{3} u_{i+1}^{n+1}=A_{4} u_{i-1}^{n}+A_{5} u_{i}^{n}+A_{6} u_{i+1}^{n}+12 P k
$$

Where

$$
\begin{aligned}
& A_{1}=-6 r+2+R k, \\
& A_{2}=12 r+8+4 R k, \\
& A_{3}=-6 r+2+R k, \\
& A_{4}=6 r+2-R k, \\
& A_{5}=-12 r+8-4 R k, \\
& A_{6}=6 r+2=R k
\end{aligned}
$$

When a similar procedure is applied to equation (10), we obtain $B_{1} \theta_{i-1}^{n i 1}+B_{2} \theta_{i}^{n+1}+B_{3} \theta_{i+1}^{n+1}=B_{4} \theta_{i-1}^{n}+B_{5} \theta_{i}^{n}+B_{6} \theta_{i+1}^{n}+12 k Q P_{r}$

Where

$$
\begin{aligned}
& B_{1}=2 P_{r}-6 r-8 R r+S k P_{r}, \\
& B_{2}=8 P_{r}+12 r+16 R r+4 S k P_{r}, \\
& B_{3}=2 P_{r}-6 r-8 R r+S k P_{r}, \\
& B_{4}=2 P_{r}+6 r+8 R r-S k P_{r}, \\
& B_{5}=8 P_{r}-12 r-16 R r-4 S k P_{r}, \\
& B_{6}=2 P_{r}+6 r+8 R r-S k P_{r}
\end{aligned}
$$

When a similar procedure is applied to equation (11), we obtain
$E_{1} C_{i-1}^{n+1}+E_{2} C_{i}^{n+1}+E_{3} C_{i+1}^{n+1}=E_{4} C_{i-1}^{n}+E_{5} C_{i}^{n}+E_{6} C_{i+1}^{n}+S_{r} \frac{\partial^{2} \theta}{\partial y^{2}} 12 k S_{c}$

Where

$$
\left.\begin{array}{l}
E_{1}=-6 r+2 S_{c}+K_{r} S_{c} k, \\
E_{2}=12 r+8 S_{c}+4 K_{r} S_{c} k, \\
E_{3}=-6 r+2 S_{c}+K_{r} S_{c} k, \\
E_{4}=6 r+2 S_{c}-K_{r} S_{c} k, \\
E_{5}=-12 r+8 S_{c}-4 K_{r} S_{c} k, \\
E_{6}=6 r+2 S_{c}-K_{r} S_{c} k
\end{array}\right\}
$$

Here, $r=\frac{k}{h^{2}}$, while $k, h$ are the sizes of mesh in the direction along $y$ and time-direction, with indices $I$ and $J$ referring to space and time respectively. The mesh system consists of $\mathrm{h}=0.1$ and $\mathrm{k}=0.001$. In the equations (11), (12) and (13) considering $i=1$ in (1) and employing the boundary conditions (10), we obtain the subsequent system equations: $A_{i} X_{i}=B_{i}$ for $i=1(1) n$ Where $A_{i}$ 's are matrices of order n and $X_{i}, B_{i}$ 's are column matrices with n components. Thomas algorithm employed to acquire the relevant solutions with regard to the system of equations considered above for the aspects of velocity, temperature, and concentration. Besides the numerical solutions can be acquired for the

\begin{tabular}{|c|c|c|c|}
\hline \multirow[b]{2}{*}{$\mathrm{y}$} & \multicolumn{3}{|c|}{$\mathrm{u}$} \\
\hline & $\begin{array}{c}\text { Size of Mesh } \\
\mathrm{k}=0.001 \\
\mathrm{~h}=0.1\end{array}$ & 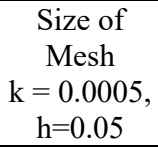 & $\begin{array}{c}\text { Size of Mesh } \\
k=0.00025 \\
h=0.025\end{array}$ \\
\hline 0 & 0 & 0 & 0 \\
\hline 0.5 & 0.364470 & 0.364025 & 0.363979 \\
\hline 1 & 0.280719 & 0.280738 & 0.280831 \\
\hline 1.5 & 0.155177 & 0.155504 & 0.155662 \\
\hline 2 & 0.074048 & 0.074378 & 0.074514 \\
\hline 2.5 & 0.031897 & 0.032127 & 0.032218 \\
\hline 3 & 0.012464 & 0.012601 & 0.012653 \\
\hline 3.5 & 0.004401 & 0.004473 & 0.004499 \\
\hline 4 & 0.001398 & 0.001431 & 0.001443 \\
\hline 4.5 & 0.000398 & 0.000411 & 0.000416 \\
\hline 5 & 0.000101 & 0.000105 & 0.000107 \\
\hline
\end{tabular}
considered system of equations by running the same on MATLAB. MATLAB has been employed with reduced values of ' $h$ ' and ' $k$ ' to establish the convergence and stability pertaining to the the method of Galerkin finite element, where considerable variations in the values of $u$, $\mathrm{T}$ and $\mathrm{C}$ have not been perceived. Consequently, it can be established that the method of Galerkin finite element is stable and convergent.

\section{Study of grid independence:}

To analyze grid independency/dependency it is better to test the solution for several mesh sizes and get a range at which there is no variation in the solution. The mathematical estimation of velocity for specific inputs of mesh(grid) size at time $t=0.2$, are shown in the above Table 1 . From this table, we observed that, variation of velocity is closer for various mesh size at time $\mathrm{t}=0.2$. Hence, we conclude that, the computational results are stable and converge.

\section{Table 1:}

The numerical values of $\mathrm{u}$ for variation of mesh (Grid) sizes (when $P r=0.71, K=0.5, M=2, E c=0.1, S c=0.22, G m=3, G r=3, K r=1.0, R=2$, $S=2, S r=2$ ). 


\section{RESULT AND DISCUSSION}

This current study addresses the issues pertaining to an unsteady free convection flow of mass transfer considering the viscous dissipation of a fluid past an infinite vertical permeable plate. Innumerable numerical computations have been applied for the velocity and the non -dimensional, temperature, and concentration ' $\mathrm{C}$ ', for various parameter indicated below:

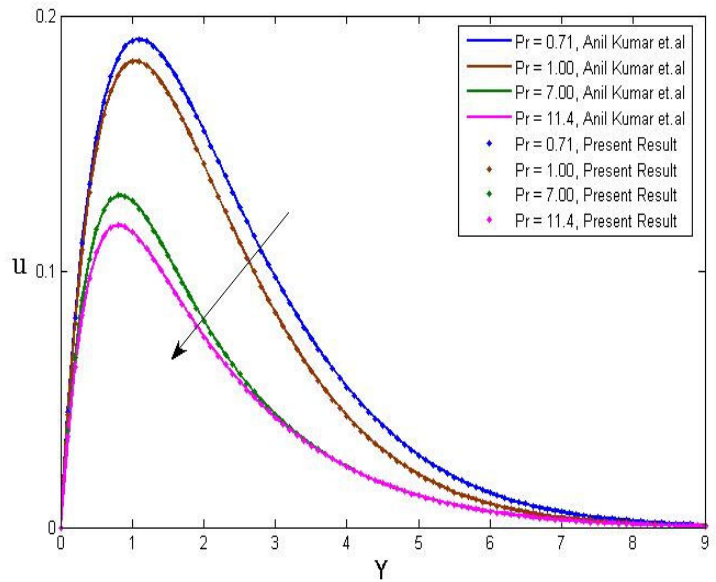

Fig. 2 Profile of Velocity for diverse values of $\operatorname{Pr}$ when $K=0.5, M=2$, $E c=0.1, S c=0.22, G m=3, G r=3, K r=1.0, R=2$.

It can be perceived from Fig. 2, that the profile of velocity decreases corresponding to the increase in Prandtl number $\mathrm{Pr}$. This study makes a comparative analysis with the available previous studies without the source of heat and the Soret effect for establishing the accuracy and validity of the output numerical results. The profiles of velocity for diverse values of $\operatorname{Pr}$ have been studied in comparison with the available solutions proposed by Anil Kumar et al. (2018) and found to largely concur. It is further perceived that the profile of velocity decreases corresponding to the increase in $P r$. This could be consequent to the fact that fluids having greater $\operatorname{Pr}$ contain higher levels of viscosities causing a reduction in velocities. Fig. 3 illustrates that the profile of velocity decreases corresponding to the increase in Prandtl number Pr. It is further perceived that the profile of velocity decreases corresponding to the increase in $P r$. This could be consequent to the fact that fluids having greater $\mathrm{Pr}$ contain higher levels of viscosities causing a reduction in velocities.

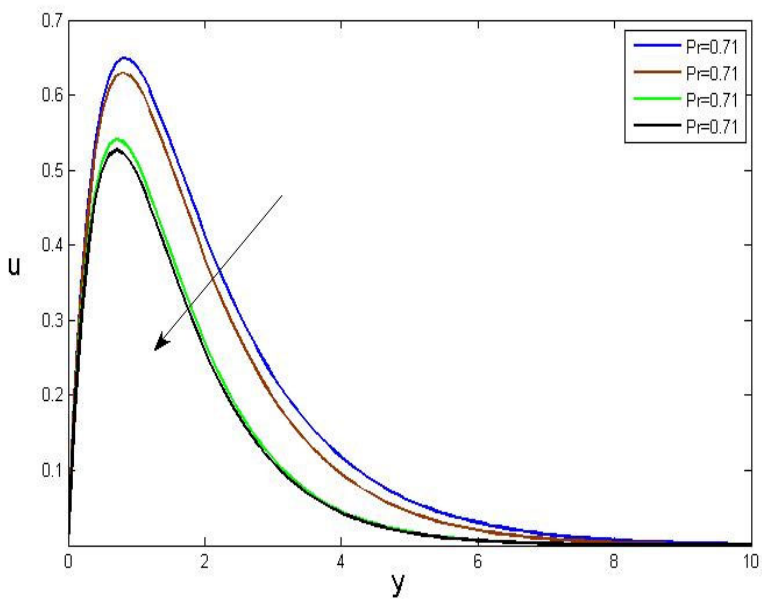

Fig. 3 Profile of Velocity for diverse values of $\operatorname{Pr}$ when $K=0.5, M=2$, $E c=0.1, S c=0.22, G m=3, G r=3, K r=1.0, R=2, S=2, S r=2$.

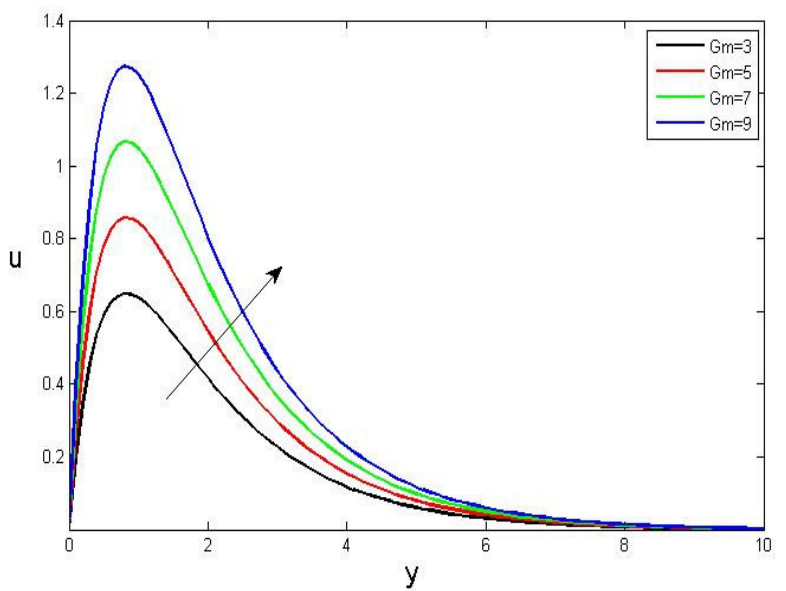

Fig. 4 Profile of Velocity for diverse values of $G m$ when $\operatorname{Pr}=0.71, M=2$, $E c=0.1, S c=0.22, G r=3, R=2, K=0.5, K r=1.0, S=2, S r=2$.

It is evident from Fig. 4 that the velocity increases corresponding to the increase in Grashof number $\mathrm{Gm}$. The ratio between the viscous and buoyancy forces operating on a fluid is approximated through Modified Grashof number, where any increase of $\mathrm{Gm}$ results in a corresponding increase in the forces of buoyancy besides a corresponding decrease in the viscous forces. In instances of decrease of viscosity, the fluid's internal resistance decreases leading to an automatic increase in the fluid velocity. The ratio between the forces of viscosity and buoyancy operating on a fluid is approximated through Grashof number $G r$. Here, any increase of $G r$ results in a corresponding increase in the forces of buoyancy besides a corresponding decrease in the viscous forces as depicted in Fig. 5. In instances of decrease of viscosity, the fluid's internal resistance decreases leading to an automatic increase in the fluid velocity. Fig. 6 illustrates the profile of velocity for diverse values of the parameter for porosity $K$. In the characterization of the properties of transport of mass and heat in a porous medium, the very factor of permeability stands as a prominent parameter. It is evident that there is an increase in $K$ in proportion to the increase in the velocity, as the increased permeability of the medium indicates decreased resistance owing to the presence of the porous matrix within the medium

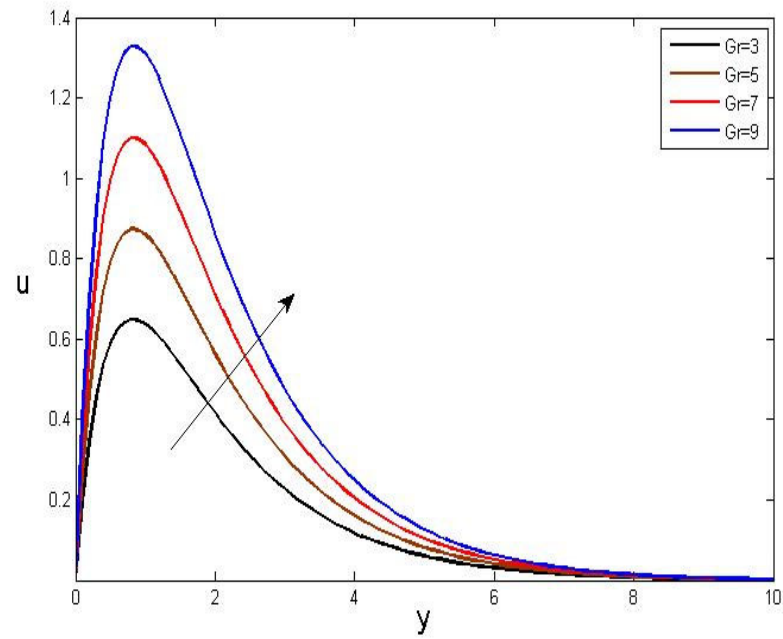

Fig. 5 Profile of Velocity for diverse values of $G r$ when $\operatorname{Pr}=0.71, M=2$, $E c=0.1, S c=0.22, G m=3, R=2, K=0.5, K r=1.0, S=2, S r=2$. 


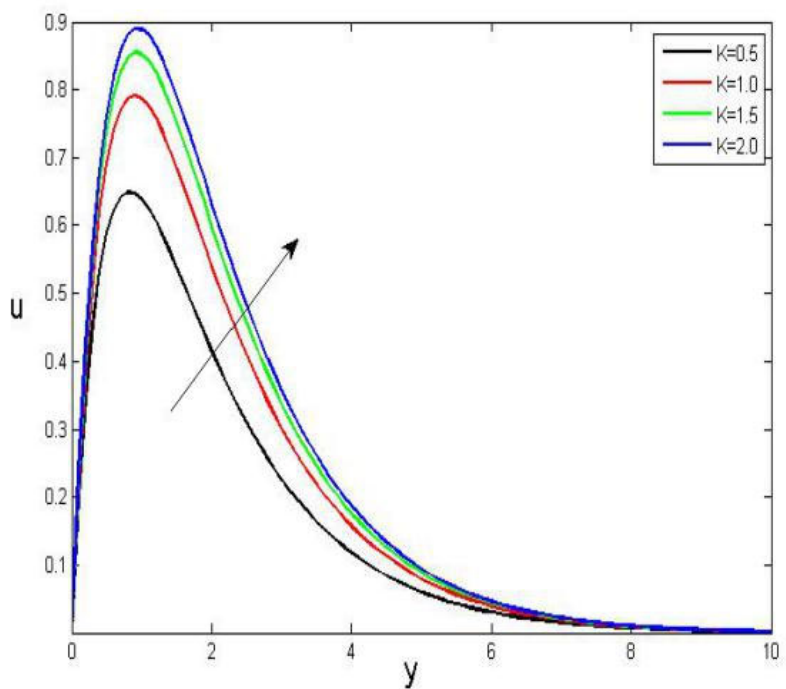

Fig. 6 Profile of Velocity for various values of $K$ when $P r=0.71, M=2$, $E c=0.1, S c=0.22, G m=3, R=2, G r=3, K r=1.0, S=2, S r=2$.

Fig. 7 illustrates the differences in the profile of velocity in conjunction with the Magnetic field parameter $M$. It is evident that increasing the Magnetic field results in a corresponding increase in the magnetic strength and the mass of the fluid particle causing the corresponding decrease in velocity. Consequent to the impact of the transverse magnetic field on a fluid that conducts electrically, there is a considerable increase in a resistive sort of force known as Lorentz force, which almost acts as a drag force. Any increase in the value of $\mathrm{M}$ leads to a corresponding force of resistance. Fig. 7. has been plotted in such a way as to illustrate the differences in the profile of velocity corresponding to $M$. It is evident that any increase in $M$ results in a proportionate increase in the magnetic strength while slowing down the fluid motion. Fig. 8 illustrates the profile of temperature for diverse values of Eckert number Ec. The Ec represents the interrelationship of the flow's kinetic energy and enthalpy. This represents the energy transformation from kinetic to internal in terms of work done against the stress of the viscous fluid. This energy manifests in the form of heat during dissipation. Consequently, the dissipative heat triggers an increase in temperature.

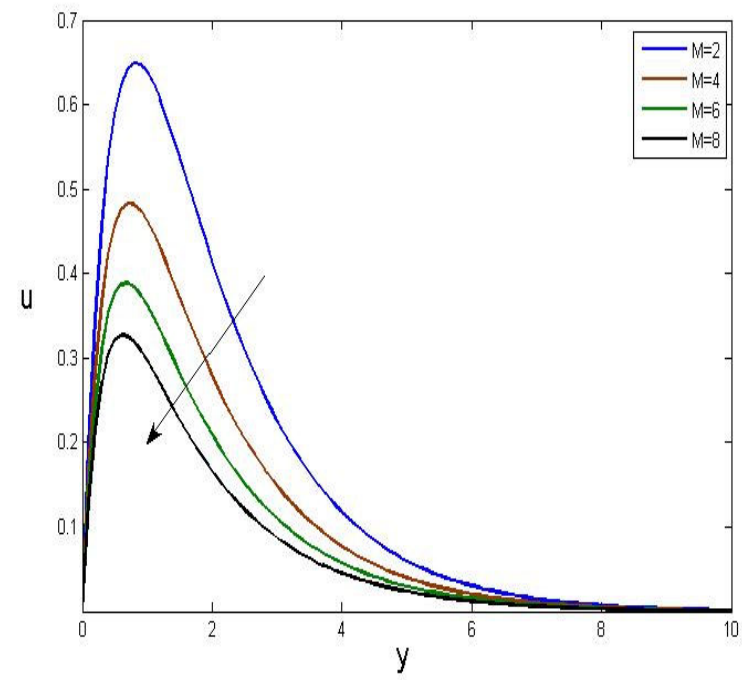

Fig. 7 Profile of Velocity for diverse values of $M$ when $K=0.5, \operatorname{Pr}=0.71$, $E c=0.1, S c=0.22, G m=3, R=2, G r=3, K r=1.0, S=2, S r=2$.

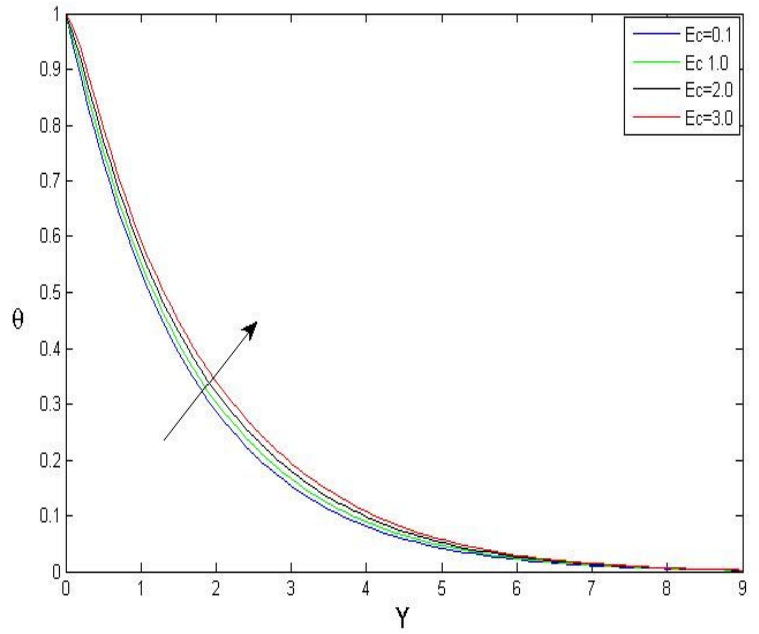

Fig. 8 Profile of Temperature for various values of $E c$ when $K=0.5$, $M=2, P r=0.71, S c=0.22, G m=3, R=2, G r=3, K r=1.0, S=2, S r=2$.

Fig. 9 depicts the profile of temperature for diverse values of the parameter of Radiation $R$. Any increase in $R$ increases the viscosity of the fluid, finally resulting in the decrease both in the temperature and the thickness of the thermal boundary layer. The results as depicted in Fig. 10 illustrate an increase in the value of the Prandtl number $\operatorname{Pr}$ causing a corresponding decrease in the field of temperature. Besides, any rise in $\operatorname{Pr}$ causes a corresponding decrease both in the temperature and the thickness of the thermal boundary layer. Fig. 11 illustrates the rise in the value of the Heat Source Parameter $S$ correspondingly decreasing the boundary layer as can be expected. This is because the absorption of heat automatically reduces the force of buoyancy which in turn slows down the rate of the flow. Fig. 12 reflects the profile of concentration for diverse values of Schmidt number $S c$. The $S c$ approximates the ratio between the mass diffusivity and kinematic viscosity. Any increase in $S c$ results in a corresponding increase in the viscous forces. The $S c$ represents the interrelationship and ratio between the momentum and mass diffusivity. The rise in $S c$ reduces the concentration correspondingly causing the effect of concentration buoyancy, thereby reducing the fluid velocity considerable.

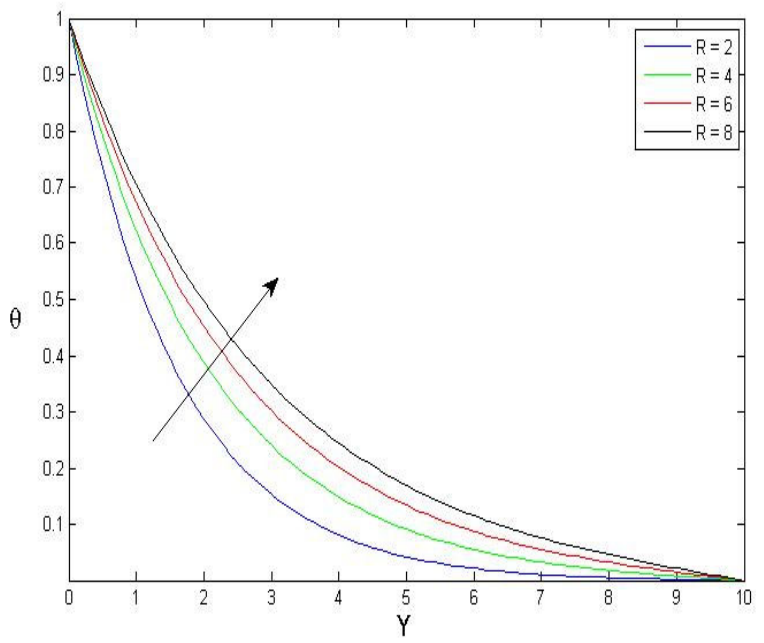

Fig. 9 Profile of Temperature for diverse values of $R$ when $K=0.5, M=2$, $E c=0.1, S c=0.22, G m=3, P r=0.71, G r=3, K r=1.0, S=2, S r=2$. 


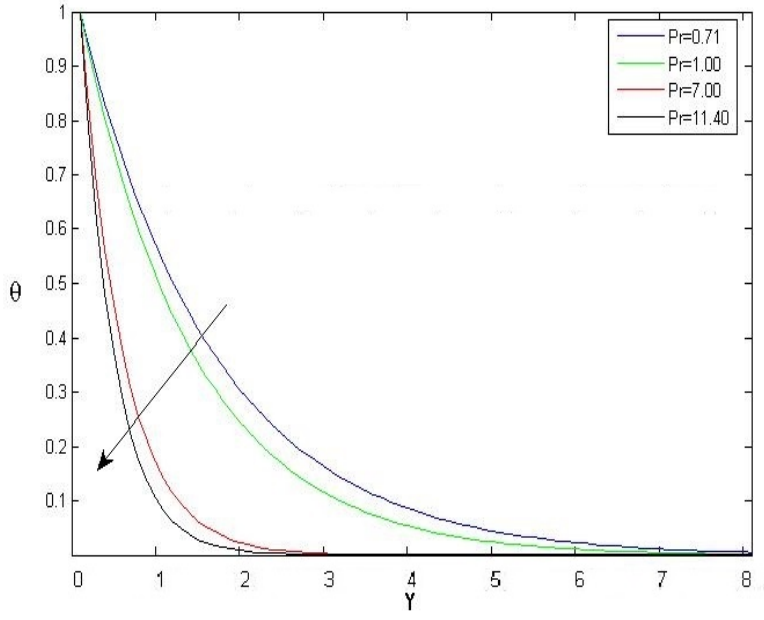

Fig. 10 Profile of Temperature for diverse values of $\operatorname{Pr}$ when $K=0.5$, $M=2, E c=0.1, S c=0.22, G m=3, G r=3, K r=1.0, R=2, S=2, S r=2$.

Fig. 13 illustrates the effect of Soret Number $S r$ on diverse profiles of concentration. This graph considers $S r$ values as $S r=2,4,6,8$. It is evident from the graph that any increase in the values of $S r$ causes a corresponding increase in the concentration profiles uniformly at every point in the field of flow. This phenomenon occurs because the diffusive species having greater $S r$ values tend to enhance the profile of concentration. Hence, conclusions can be drawn that the $\mathrm{Sr}$ values considerably influence the concentration distributions. Fig. 14 depicts the influence of the parameter of chemical reaction $\mathrm{Kr}$ on the profiles of concentration. It is obvious that an increase in $K r$ results in the reduction of concentration. Fig. 15 illustrates the Skin-friction $\tau$ in the context of time ' $t$ ' for diverse parameter values. Any increase in $M$ causes a corresponding increase in the $\tau$. The corresponding increase in the $\tau$ along with $\mathrm{M}$ can be attributed to the increased Lorentz force, which brings in supplementary momentum within the boundary layer. In contrast, an increase in $K, G m$ and $G r$ leads to a corresponding reduction in the $\tau$. The extent of $\tau$ for $P r=0.71$ is low in comparison to that of $\operatorname{Pr}=7$.

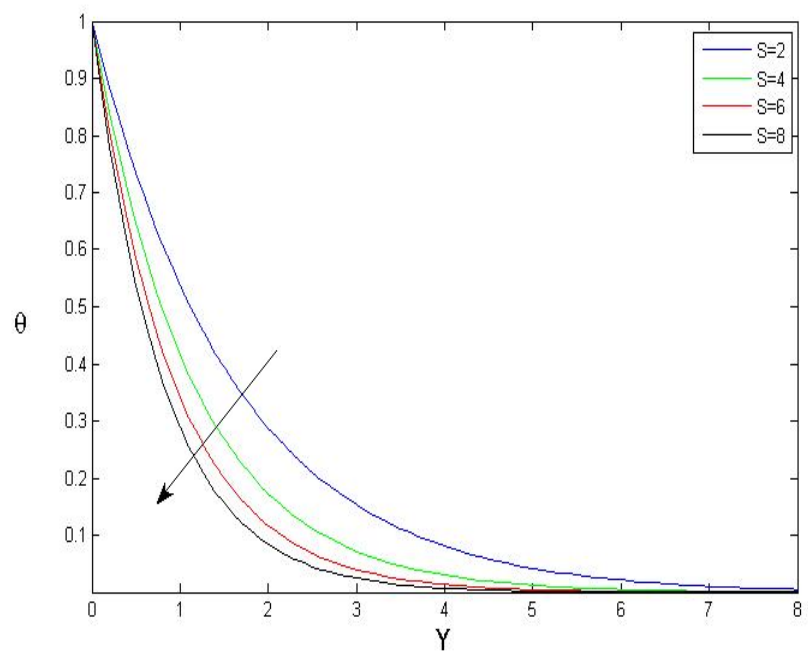

Fig. 11 Profile of Temperature for diverse values of $S$ when $K=0.5$, $M=2, E c=0.1, S c=0.22, G m=3, P r=0.71, G r=3, K r=1.0, S r=2$

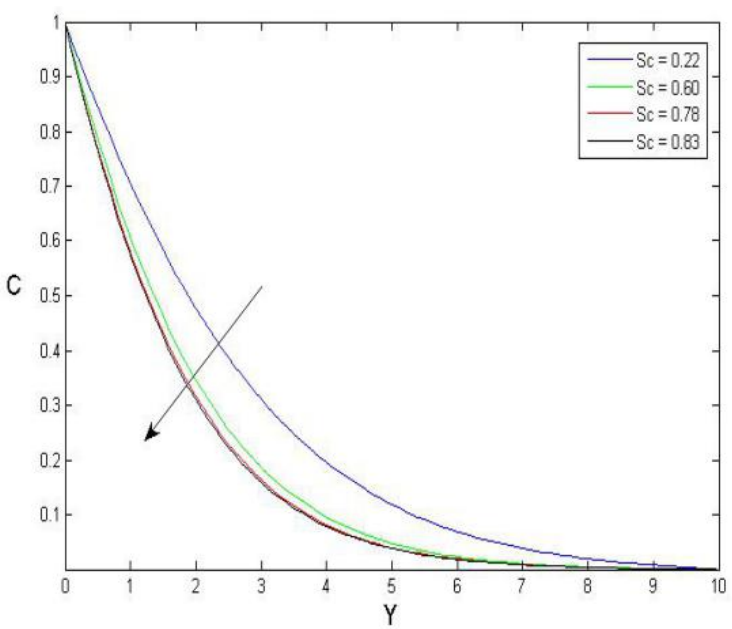

Fig. 12 Profile of Concentration for diverse values of Sc when $K=0.5$, $M=2, E c=0.1, P r=0.71 G m=3, R=2, G r=3, K r=1.0, S=2, S r=2$.

Fig. 16 illustrates a perceivable increase in the concentration transfer rate corresponding to the rising values of $S c$, and $K r$, whereas the same is found to decrease when the $S r$ value is increased. Fig. 17 illustrates the Nusselt number $N u$ along with the time ' $t$ ' for different values of metrics such as $P r, R, E c$, and $S$. Nu for $P r=7$ (in this case for water) is found to be greater than the number for $P r=0.71$. This is because the lower $P r$ values are equivalent to correspondingly enhancing the thermal conductivities. Consequently, the heat can more swiftly diffuse further away from the plate than with higher $P r$ values. The same applies to $S$ also. Hence, an increased heat transfer rate can be perceived. It can also be perceived that there is a considerable fall in the heat transfer rate corresponding to the rise in $R$ and $E c$.

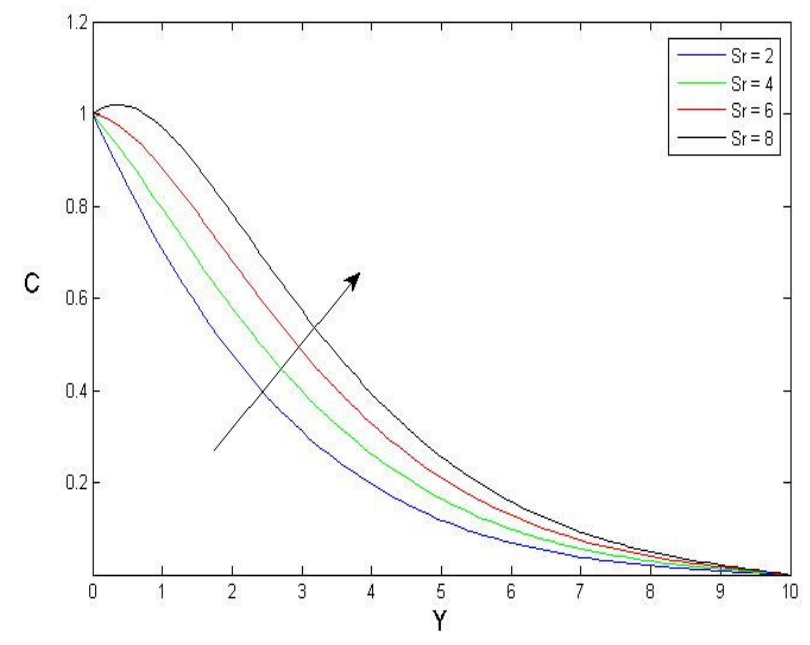

Fig. 13 Profile of Concentration for diverse values of $\mathrm{Sr}$, when $K=0.5$, $M=2, E c=0.1, P r=0.71 G m=3, R=2, G r=3, S c=0.22, \mathrm{~S}=2$. 


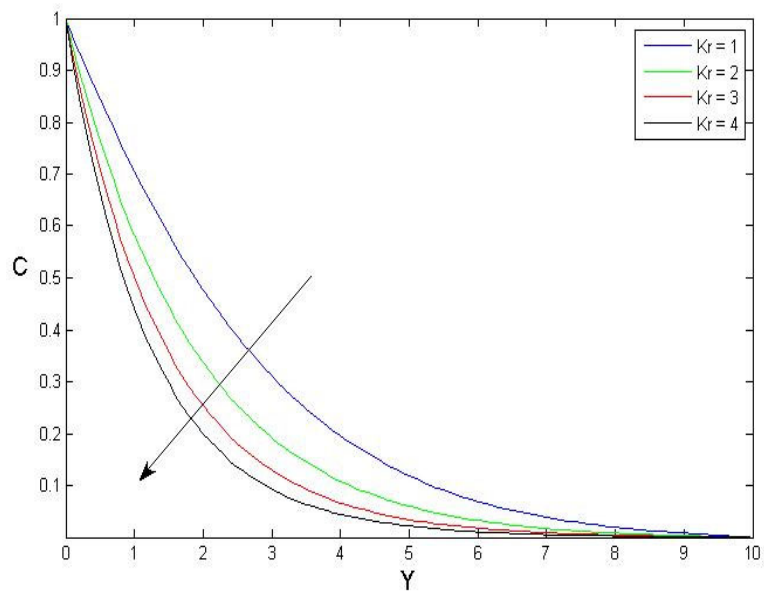

Fig. 14 Profile of Concentration for diverse values of $K r$ when $K=0.5$, $M=2, E c=0.1, P r=0.71 G m=3, R=2, G r=3, S c=0.22, S=2, S r=2$.

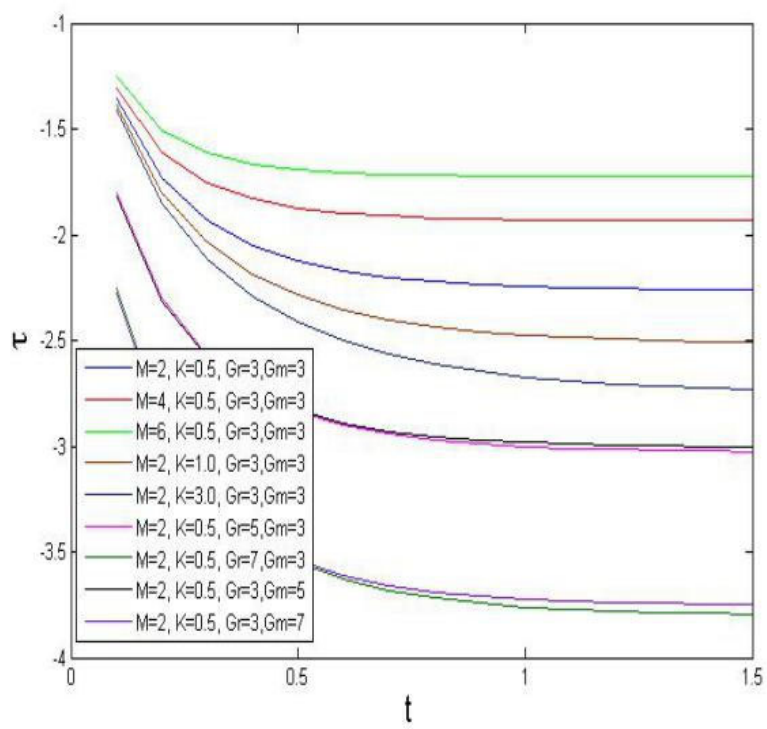

Fig. 15 Profile of Skin Friction for diverse values of $M, K, G r$, and $G m$.

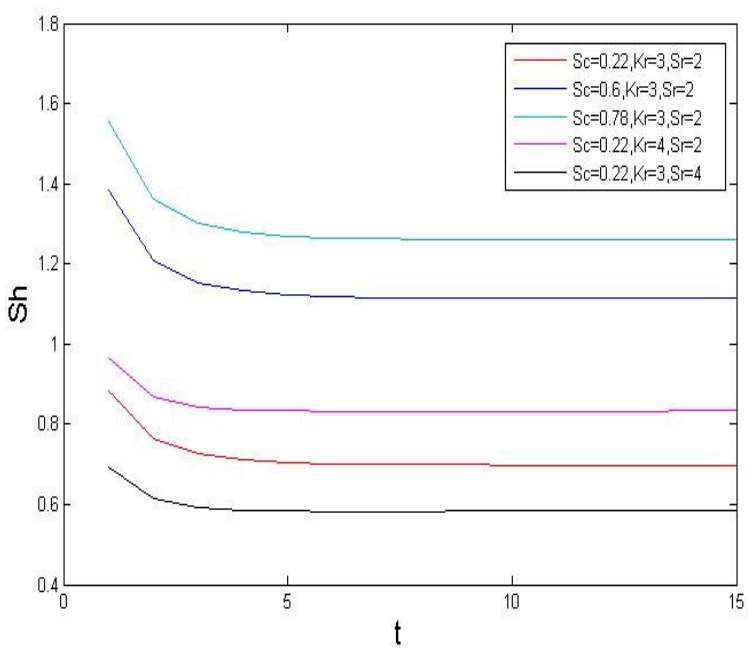

Fig. 16 Profile of the Sherwood number for diverse values of $S c, K r$, and Sr.

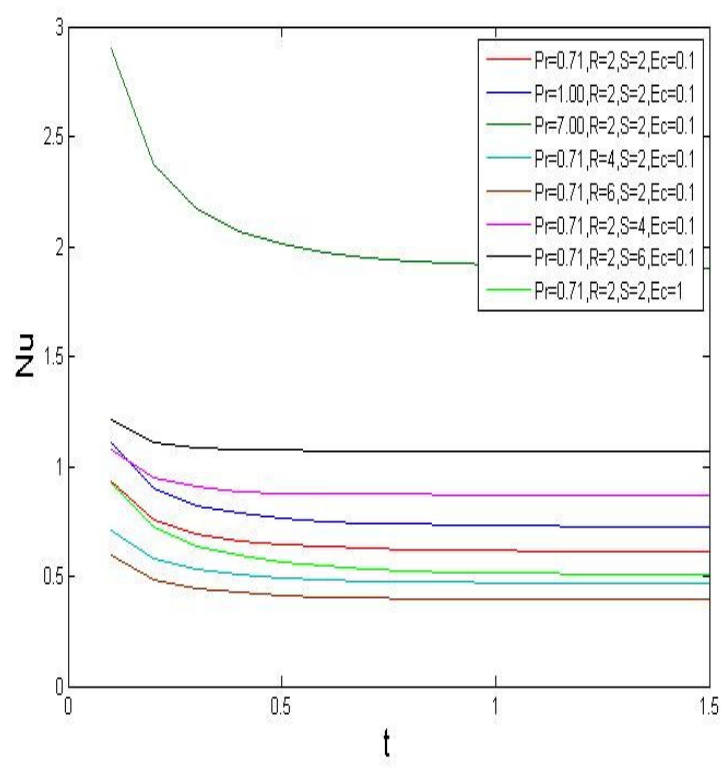

Fig. 17 Profile of the Nusselt number for diverse values of $\operatorname{Pr}, R, S$, and Ec.

\section{CONCLUSIONS}

In this study, an attempt has been made to analyze numerically the impact of viscous dissipation of thermal radiation on the diffusion flow of mass and heat of MHD past an infinite vertical plate which has been embedded in a permeable medium containing variable conditions of the surface. The method of Galerkin finite element is employed for solving the equations which governs the flow. The following are some of the interesting outcome factors of the study about the physical aspects of concentration, temperature, and the velocity of the flow:

- Velocity increases corresponding to the enhancement in the $\mathrm{Gr}$ and $G m$ numbers.

- Velocity decreases corresponding to the enhancement in the magnetic parameter $M$ and the permeable nature of the porous medium $K$.

- An increase in $S c$ and time $t$ causes a decrease in the velocity.

- $\quad$ The temperature rises corresponding to the rise in $E c$ as well as time $t$, but reduces with the rise in the parameter of radiation $R$.

- An increase in $S c$ causes a corresponding decrease in the level of concentration.

- Greater $S r$ values tend to enhance the level of concentration.

- A rise in $M$ causes an increase in the Skin-friction $\tau$.

- Increased $K, G m$, and $G r$ cause a corresponding decrease in the $\tau$.

- A rise in the values of $S c$ correspondingly increases the Sherwood number $S h$.

- Increasing $M$ causes a corresponding increase in the $\tau$.

- Increasing the values of $K, G m$ and $G r$ causes a corresponding decrease in the $\tau$.

- $\quad$ Enhanced values of $S c$ cause a corresponding rise in the $S h$.

- $\quad$ Enhanced values of $S r$ cause a corresponding decrease in the Sh.

- $\quad$ Rise in the values of $\operatorname{Pr}$ and $S$ result in a corresponding increase in $N u$. 


\section{NOMENCLATURE:}

$C_{\infty}^{\prime} \quad$ Concentration of the fluid far away from the plate $\left(\mathrm{Kg} \mathrm{m}^{-3}\right)$

$C_{w}^{\prime} \quad$ Concentration of the plate $\left(K g \quad m^{-3}\right)$

$y \quad$ Dimensionless displacement $(m)$

$T_{\infty}^{\prime} \quad$ Fluid temperature away from the plate $(K)$

Gm Grashof number for mass transfer

Gr Grashof number for heat transfer

$u \quad$ Non-dimensional fluid velocity $\left(\mathrm{ms}^{-1}\right)$

Sh The local Sherwood number

$u^{\prime} \quad$ Velocity component in $x^{\prime}-$ direction $\left(m s^{-1}\right)$

$g \quad$ Acceleration of gravity, $9.81\left(\mathrm{~m} \mathrm{~s}^{-2}\right)$

$x^{\prime} \quad$ Coordinate axis along the plate $(m)$

Re Reynolds number

$D \quad$ Solute mass diffusivity $\left(m s^{-2}\right)$

$\mathrm{Nu}$ The local Nusselt number

$\tau \quad$ Skin Friction

$B_{0} \quad$ Uniform magnetic field (Tesla)

$H_{0} \quad$ Magnetic Induction

$\rho \quad$ The constant density $\left(\begin{array}{ll}K g & m^{-3}\end{array}\right)$

$\beta \quad$ Volumetric coefficient of thermal expansion $\left(K^{-1}\right)$

$y^{\prime} \quad$ Co-ordinate axis normal to the plate $(m)$

Ec Eckert number

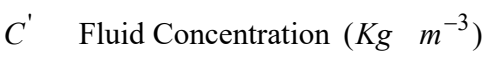

$T_{w}^{\prime} \quad$ Fluid temperature at the wall $(K)$

$T^{\prime} \quad$ Fluid temperature $(K)$

$M \quad$ Magnetic parameter

Pr Prandtl number

$q_{r} \quad$ Radiative heat transfer coefficient $S c \quad$ Schmidt number

$C_{p} \quad$ Specific heat at constant Pressure $\left(J K^{-1} K\right)$

$v \quad$ Kinematic viscosity $\left(m^{2} s^{-1}\right)$

$\mu_{0} \quad$ Magnetic Permeability $\left(N . A^{-2}\right)$

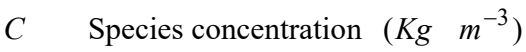

\section{Greek Symbols:}

$K \quad$ Thermal conductivity of the fluid $\left(\mathrm{Wm}^{-1} \mathrm{~K}^{-1}\right)$

$\theta \quad$ Non dimensional fluid temperature $(K)$

$\beta^{*} \quad$ Volumetric Coefficient of thermal expansion with concentration $\left(m^{-3} \mathrm{Kg}\right)$

$\sigma \quad$ Electric conductivity of the fluid $\left(\mathrm{s} \mathrm{m}^{-1}\right)$

\section{Superscripts:}

‘ Dimensionless Properties

\section{Subscripts:}

$\infty \quad$ Free stream conditions

p Plate

\section{ACKNOWLEDGEMENTS:}

The investigators remain profusely grateful to Koneru Lakshmaiah Education Foundation, Vijayawada for extending support and assistance with required permissions during this research study at the Department of Mathematics and to Rise Krishna Sai Group of Institutions, Ongole, for necessary permissions and extending facilities for the work.

\section{REFERENCES}

Agrawal H, Ram P.C and Singh V. 1984. "Effects of Hall Currents on Hydro-Magnetic Free Convection with Mass Transfer in a Rotating Fluid." Astrophysics Space Science, 100: 297-283.

https://doi.org/10.1007/BF00651602

Alam M. M., Alim M. A. and Chowdhury M. M. K. 2007. "Viscous Dissipation Effects on MHD Natural Convection Flow over a Sphere in the Presence of Heat Generation." Nonlinear Analysis Modeling and Control, 12:447-459.

Alim M. A., Rahman M. M. and Aktar S. 2008. "Viscous Dissipation Effects on Natural Convection Flow along a Sphere with Radiation Heat Loss." BSME-ASME International Conference on Thermal Engineering, Dhaka, Bangladesh.

https://doi.org/10.3329/jname.v10i1.12809

Anil Kumar M., Dharmendar Reddy Y. and Srinivasa Rao V. 2018. "Effect of Thermal Radiation and Chemical Reaction on Unsteady MHD Free Convection Flow of an Incompressible Fluid Past an Infinite Vertical Porous Plate in Presence of Viscous Dissipation." International Journal of Management Technology and Engineering, 8: 43-58, https://doi.org/16.10089/IJMTE.2018.V8112.17.2005

Astuti .H, Sri .P and Kaprawia .S. 2019 "Natural Convection of Nano fluids Past an Accelerated Vertical Plate with Variable Wall Temperature by Presence of the Radiation." Frontiers in Heat and Mass Transfer (FHMT): 13, 3.

https://dx.doi.org/10.5098/hmt.13.3

Bhaskara Reddy N and Bathaiah D. 1982. "MHD Combined Free and Forced Convection Flow through Two Parallel Porous Walls." Acta Mechanica, 42, 239-251.

https://doi.org/10.1007/BF01177195

Bhaskara Reddy N. and Bathaiah D. 1981. "Magneto Hydrodynamic Free Convection Laminar Flow of an Incompressible Viscid Elastic Fluid.” Reg. J. of Energy Heat Mass Transfer 3(4): 239- 255.

Crepeau J. C. and Clarksean R., 1997 "Similarity Solutions of Natural Convection with Internal Heat Generation Transactions of ASME." Journal of Heat Transfer 119: 184-185.

https://doi.org/10.1115/1.2824086

Elabashbeshy E.M.A. 1997. "Heat and Mass Transfer along a Vertical Plate with Variable Temperature and Concentration in the Presence of Magnetic Field." Int. J. Eng. Sci 34: 515-522. https://doi.org/10.1016/S0020-7225(96)00089-4

Gehart B. and Pera L. 1971. "The Nature of Vertical Natural Convection Flows Resulting from the Combined Buoyancy Effects of Thermal and Mass Diffusion." International Journal of Heat Mass Transfer 14: 20252050.

https://doi.org/10.1016/0017-9310(71)90026-3

Jang J.Y and Ni J.R., 1989, "Transient Free Convection with Mass Transfer from an Isothermal Vertical Plate Embedded in a Porous Medium." Int. J. Heat and Mass Transfer, 10: 59-65 
Jha B.K. 1991. "MHD Free Convection and Mass Transfer Flow through a Porous Medium." Astrophysics Space Science, 175: 283-289.

https://doi.org/10.1007/BF00644290

Korycki R. 2006. "Sensitivity Analysis and Shape Optimization for Transient Heat Conduction with Radiation." Int. J. Heat Mass Transfer, 49:2033-2043.

https://doi.org/10.1016/j.ijheatmasstransfer.2006.01.007

Loganathan P. and Arasu P. P. 2010. "Thermophoresis Effects on NonDarcy MHD Mixed Convective Heat and Mass Transfer Past a Porous Wedge in the Presence of Suction/Injection." Theoretic Applied Mechanics, 37(3), 203-227.

https://doi.org/10.2298/TAM1003203L

Middleman S., 1998, “An Introduction to Mass and Heat Transfer,” John Wiley \& Sons, Inc.,

Nagalakshmi P.S.S and Vijaya N. 2020. "MHD flow of Carreau Nano fluid explored using CNT over a nonlinear elongated sheet" Frontiers in Heat and Mass Transfer (FHMT), 14, 4.

https://dx.doi.org/10.5098/hmt.14.4

Pal D. and Talukdar B. 2012. "Influence of Fluctuating Thermal and Mass Diffusion on Unsteady MHD Buoyancy-Driven Convection Past a Vertical Surface with Chemical Reaction and Soret Effects." Comm. Nonlinear Sci. Numer. Simulat., 17(4), 1597-1614.

https://doi.org/10.1016/i.cnsns.2011.08.038

Prabhakar Reddy B., 2016, "Mass Transfer Effects on an Unsteady MHD Free Convective Flow of an Incompressible Viscous Dissipative Fluid
Past an Infinite Vertical Porous Plate." Int. J. of Applied Mechanics and Engineering, 21(1), 143-155.

https://doi.org/10.1515/ijame-2016-0009

Reddy, B. P. and Rao, J. A. 2011. "Radiation and Thermal Diffusion Effects on An Unsteady MHD Free Convection Mass-Transfer Flow Past an Infinite Vertical Porous Plate with The Hall Current and a Heat Source.” J. Eng. Phys. Thermophys., 84(6), 1369-1378. https://doi.org/10.1007/s10891-011-0607-4

Rubin H. and Atkinson J. 2001. "Environmental Fluid Mechanics." Marcel Dekker Inc, New York.

Salina A., Mahmuda Binte Mostofa Ruma and N. Parveen., 2010 "Viscous Dissipation Effects on Natural Convection Flow along a Sphere with Heat Generation." Global Journal of Science Frontier Research, 107-14.

https://journalofscience.org/index.php/GJSFR/article/view/29

Samad M.A., Karim M.E. and Mohammad D. 2010. "Free Convection Flow through a Porous Medium with Thermal Radiation, Viscous Dissipation and Variable Suction in the Presence of Magnetic Field." Bangladesh Journal of Scientific Research, 23(1), 61-72. https://doi.org/index.php/BJSR/article/view/8999

Soundalgekar V.M. and Wavre P.D. 1977. "Unsteady Free Convection Flow Past Infinite Vertical Plate with Constant Suction and Mass Transfer." - Int. J. Heat and Mass Transfer, 19: 1363-1373. https://doi.org/10.1016/0017-9310(77)90033-3 